\title{
Avaliação de Três Formas de Soluções de Sistemas de Equações de Transporte Advectivo-difusivo com Decaimento
}

N.M.L. ROMEIRO ${ }^{1}$, Depto de Matemática, CCE, UEL, Campus Universitário, Cx.P. 6001, 86051-970 Londrina, PR, Brasil.

R.G.S. CASTRO ${ }^{2}$, Laboratório de Ciências Matemáticas, CCT, UENF, Av. Alberto Lamego, 2000, 28013-602 Campos dos Goytacazes, RJ, Brasil.

L. LANDAU ${ }^{3}$, Programa de Engenharia Civil, COPPE Centro de Tecnologia, UFRJ, Bloco B/sala 100, 21945-970, Rio de Janeiro, RJ, Brasil.

Resumo. Este trabalho apresenta uma avaliação abrangente de três formas para resolver sistemas de equações de transporte advectivo-difusivo com decaimento em um meio fluido. As formas avaliadas diferenciam-se em acoplada implícita, desacoplada sequencialmente implícita e desacoplada explícita. Os modelos podem ser descritos por esquemas de reações irreversível ou reversível envolvendo tanto os processos de reações lineares quanto os não lineares. Para o caso das equações que envolvam processos não lineares pode-se utilizar a primeira ordem da expansão em série de Taylor de forma a lineariza-los, sendo então possível analisar este caso também. Avaliou-se os resultados obtidos considerando o número da Peclet de malha (Pe). Se $P e>1$, a advecção é dominante, os sistemas acoplado implícito, desacoplado sequencialmente implícito e desacoplado explícito geram resultados satisfatórios. Observou-se ainda nas avaliações que nos sistemas acoplado e desacoplado sequencialmente implícito os resultados são satisfatórios para qualquer valor de Peclet. O mesmo não ocorre para o caso do desacoplamento explícito, onde se $P e<1$, difusão dominante, o método gera resultados não satisfatórios.

Palavras-chave. Equações diferenciais parciais, equações de transporte, modelo acoplado, decaimento, método dos elementos finitos.

\section{Introdução}

Tem-se desenvolvido nos últimos anos uma extensa variedade de modelos baseados em sistemas de equações de transporte advectivo-difusivo (ETAD) com decaimento devido a sua aplicabilidade em problemas de contaminação de solos, de águas e de remediação por biodegradação. Muitos destes modelos são baseados em aproximações

\footnotetext{
${ }^{1}$ nromeiro@uel.br

${ }^{2}$ sanabria@uenf.br

${ }^{3}$ landau@lamce.ufrj.br
} 
numéricas, pois estes podem empregar tanto reações cinéticas simples quanto complexas, tais como reações não lineares do tipo Monod [3, 4]. Os esquemas de soluções dos modelos que usam cinéticas de Monod geram custos computacionais adicionais devido a não linearidade e ao acoplamento quando múltiplas espécies são consideradas, mas uma das vantagens em utilizar este tipo de modelo refere-se a facilidade na calibração devido a flexibilidade introduzida via os parâmetros cinéticos. Existem diversos modelos analíticos cujos sistemas de ETAD com decaimento empregam uma cinética simplificada de primeira ordem [7, 15, 16, 5, 17]. Romeiro (2003) utilizou a primeira ordem da expansão em série de Taylor nos termos não lineares de um modelo unidimensional de ETAD de tal forma a lineariza-los [11], comparou as soluções numéricas dos modelos usando coeficientes de decaimento não lineares e linearizados e utilizou uma generalização da solução analítica para verificar a aplicabilidade do modelo linearizado [12, 13, 14]. Desta forma, os termos dos processos de reações no sistema de ETAD com decaimento podem ser descritos com coeficientes lineares de primeira ordem ou não lineares do tipo Monod. Resolvido o problema da não linearidade, deve-se observar o acoplamento no sistema, este dificulta a implementação numérica do modelo e a validação do mesmo por meio de uma solução analítica. Assim, como uma alternativa para contornar esta dificuldade, apresenta-se neste trabalho duas formas de desacoplamento: desacoplamento sequencialmente implícito (DSI) e desacoplamento explícito (DE). O DSI consiste em diagonalizar a matriz resultante do sistema de equações do modelo de reações, gerando desta forma o desacoplamento no sistema. Já o DE consiste em separar o modelo de transporte advectivo-difusivo do modelo de reações, onde a solução do modelo de transporte entra como condição inicial para resolver analiticamente o modelo de reações. Propõe-se ainda neste trabalho avaliar e comparar as soluções entre o modelo acoplado e os dois modelos desacoplados. Para verificar a aplicabilidade das formas de soluções a serem apresentadas utiliza-se diferentes valores para o coeficiente de difusão.

\section{Sistema Acoplado de Equações}

Para modelar problemas de interesse e avaliar o transporte de degradação de $n$ espécies, envolvidas em esquemas de reações, pode-se usar o sistema unidimensional acoplado de ETAD com decaimento de primeira ordem representado por

$$
\frac{\partial \mathbf{C}}{\partial t}+u \frac{\partial \mathbf{C}}{\partial x}-D \frac{\partial^{2} \mathbf{C}}{\partial x^{2}}=-\mathbf{K C}+\mathbf{\Upsilon}
$$

onde

$$
\mathbf{C}=\left[\begin{array}{c}
C_{1} \\
C_{2} \\
\vdots \\
C_{n}
\end{array}\right], \quad \mathbf{K}=\left[\begin{array}{cccc}
k_{1} & -y_{12} k_{2} & \ldots & -y_{1 n} k_{n} \\
-y_{21} k_{1} & k_{2} & \ldots & -y_{2 n} k_{n} \\
\vdots & \vdots & \ddots & \vdots \\
-y_{n 1} k_{1} & -y_{n 2} k_{2} & \ldots & k_{n}
\end{array}\right], \quad \mathbf{\Upsilon}=\left[\begin{array}{c}
\gamma_{1} \\
\gamma_{2} \\
\vdots \\
\gamma_{n}
\end{array}\right]
$$

$C_{i}$ é a concentração da $i$-ésima espécie, $k_{i}$ é a taxa de decaimento de primeira ordem da $i$-ésima especie, $y_{i j}$ é o fator de ganho da espécie $i$ em relacão a espécie $j$ e $\gamma_{i}$ 
representa o termo fonte da $i$-ésima espécie, $i, j=1,2, \ldots, n$, onde $n$ é o total de espécies transportadas, $u$ refere-se ao coeficiente de advecção e $D$ o coeficiente de difusão.

As soluções das equações do sistema (2.1), obtidas para um meio semi-infinito, são submetido as condições iniciais $C(x, 0)=C_{i n}, \quad x \geq 0$, e as condições de fronteira $C(0, t)=C_{o}$ e $C(\infty, t)=0$ para $t>0$, onde $C_{i n}=\left[C_{i n 1}, C_{i n 2}, \ldots, C_{i n n}\right]^{T}$ e $C_{o}=\left[C_{o 1}, C_{o 2}, \ldots, C_{o n}\right]^{T}$ referem-se as condições iniciais e de fronteira de cada espécie transportada.

Ressalta-se que as condições na fronteira do tipo $C(\infty, t)=0$ são utilizadas para gerar as soluções analíticas do sistema (2.1), quando este encontra-se desacoplado.

\section{Diferentes Formas para Resolver o Sistema de Equações}

O sistema de equações diferenciais parcias, representado no lado esquerdo da equação (2.1), encontra-se acoplado através dos processos das reações cinéticas de primeira ordem, incluídas no sistema de ETAD com decaimento. Alguns pesquisadores como Clement (2001), Park et al. (1996) e Kaluarachchi et al. (1995), resolvem o sistema desacoplado [5, 10, 9], outros como Farrell et al. (1998), Croucher et al. (1998), Sun et al. (1999a e 1999b) e Bai et al. (1999), resolvem acoplado [8, 6, 15, 16, 1]. Observa-se que tanto Clement (2001) quanto Kaluarachchi et al. (1995) [5, 9], resolvem o modelo desacoplado de formas diferentes, e usam soluções analíticas para resolver o modelo desacoplado. Assim, este trabalho apresenta uma avaliação entre as soluções das duas formas de desacoplamento, DSI e DE, comparando-as com as soluções do sistema acoplado.

\subsection{Forma Acoplada Implícita (AI)}

O sistema de ETAD com decaimento envolvendo $n$ espécies reativas dado em (2.1) é resolvido acoplado, usando o método dos elementos finitos (MEF) estabilizados na sua formulação semi-discreta, sujeito a condições iniciais e de fronteira. Isto é, a variável espacial é discretizada usando o método dos elementos finitos streamline - upwind/Petrov-Galerkin (SUPG), e a variável temporal é discretizada usando o método trapezoidal generalizado Crank-Nicolson que é um método implícito incondicionalmente estável com precisão de segunda ordem, [2]. Este conjunto de soluções são representados por $C_{i A I}(x, t), i=1,2, . ., n$.

\subsection{Forma Desacoplada Sequencialmente Implicita (DSI)}

A forma DSI resolve o sistema de equações dado em (2.1) desacopladamente. Para isto, diagonaliza-se a matriz $\mathbf{K}$ resultante do sistema de equações dos processos das reações, gerando, desta forma, o desacoplamento do sistema [5]. Assumindo que $\mathbf{K}$ é uma matriz quadrada com um sistema completo de autovetores obtém-se a matriz 
$\mathbf{S}$ que diagonaliza a matriz $\mathbf{K}$. Obtida a matriz $\mathbf{S}$ define-se um sistema de equações auxiliares da forma

$$
\mathbf{C}=\mathbf{S B} .
$$

Substituindo (3.1) em (2.1) e denotando $\mathbf{S}^{-\mathbf{1}} \mathbf{K S}=\hat{\mathbf{K}}$, obtém-se

$$
\frac{\partial \mathbf{B}}{\partial t}+u \frac{\partial \mathbf{B}}{\partial x}-D \frac{\partial^{2} \mathbf{B}}{\partial x^{2}}=-\hat{\mathbf{K}} \mathbf{B}+\mathbf{S}^{-1} \mathbf{\Upsilon}
$$

que é um sistema de equações similar ao sistema dado em (2.1). Entretanto, sendo $\hat{\mathbf{K}}$ uma matriz diagonal, a equação (3.2) resulta em um sistema desacoplado que pode ser resolvido numericamente usando o MEF estabilizados, ou analiticamente usando a solução apresentada por Van Genuchten (1981) [17] e descrita em (3.3)

$$
\begin{aligned}
& B_{i D S I}(x, t)=\frac{1}{2}\left(B_{i 0}-\hat{\gamma}_{i} / \hat{K}_{i}\right)\left[\exp \left(\frac{\left(u-\beta_{i}\right) x}{2 D}\right) \operatorname{erfc}\left(\frac{x-\beta_{i} t}{2(D t)^{1 / 2}}\right)\right. \\
& \left.\quad+\exp \left(\frac{\left(u+\beta_{i}\right) x}{2 D}\right) \operatorname{erfc}\left(\frac{x+\beta_{i} t}{2(D t)^{1 / 2}}\right)\right]+\frac{\hat{\gamma}_{i}}{\hat{K}_{i}}\left(1-\exp \left(\hat{K}_{i} t\right)\right) \\
& +\frac{1}{2} \frac{\hat{\gamma}_{i}}{\hat{K}_{i}} \exp \left(-\hat{K}_{i} t\right)\left[\operatorname{erfc}\left(\frac{x-\beta_{i} t}{2(D t)^{1 / 2}}\right)+\exp \left(\frac{u x}{D}\right) \operatorname{erfc}\left(\frac{x+u t}{2(D t)^{1 / 2}}\right)\right],
\end{aligned}
$$

onde $\beta_{i}=u\left(1+4 \hat{K}_{i} D / u^{2}\right)^{1 / 2}, B_{i 0}$ representa a condição de fronteira e $\hat{\gamma}_{i}$ representa o termo fonte resultante do desacoplamento, da $i$-ésima espécie. Resolvendo (3.3) para cada uma das $n$ espécies obtêm-se soluções da forma $B_{i D S I}(x, t)$ a cada passo de tempo $t$. Geradas todas estas soluções, usa-se a equação (3.1) para obter as soluções em função das variáveis originais $C_{i D S I}(x, t)$.

\subsection{Forma Desacoplada Explícita (DE)}

A forma DE refere-se a uma análise similar à apresentada por Kaluarachchi et al. (1995) [9], onde divide-se o sistema dado em (2.1) em dois sistemas: o primeiro refere-se ao sistema das equações de transporte (ET) e o segundo ao sistema das equações das reações (ER), como representados em (3.4) e (3.5), respectivamente

$$
\begin{gathered}
\frac{\partial \mathbf{C}}{\partial t}+u \frac{\partial \mathbf{C}}{\partial x}-D \frac{\partial^{2} \mathbf{C}}{\partial x^{2}}=0 \\
\frac{d \mathbf{C}}{d t}=-\mathbf{K C}+\boldsymbol{\Upsilon} .
\end{gathered}
$$

Resolvendo numericamente, usando MEF estabilizados, ou analiticamente [17] as equações de (3.4), sujeitas às condições iniciais e de fronteira, obtêm-se soluções do tipo $C_{i E T}(x, t)$, como descritas em (3.6)

$$
C_{i E T}(x, t)=\frac{1}{2} C_{i 0}\left[\operatorname{erfc}\left(\frac{x-u t}{2(D t)^{1 / 2}}\right)+\exp \left(\frac{u x}{D}\right) \operatorname{erfc}\left(\frac{x+u t}{2(D t)^{1 / 2}}\right)\right] .
$$

Quanto ao sistema de ER dado em (3.5), este pode ser resolvido usando a solução analítica, pois representa um sistema linear de equações diferenciais ordinárias, ou ainda usando um método numérico, tendo como condições iniciais o sistema de soluções obtido em (3.6), resultando em soluções do tipo $C_{i D E}(x, t)$. 


\section{Análise e Aplicação das Diferentes Formas para Resolver o Sistema}

Sistemas de ETAD com decaimento podem empregar esquemas envolvendo estruturas de reações irreversível e reversível [15]. As soluções dos modelos que empregam estes esquemas podem ser simples, para o caso de reações irreversíveis, ou complexos, caso contrário. A complexidade é gerada devido ao acoplamento do sistema, como será observado no exemplo a ser ilustrado. Apresenta-se ainda comparações entre as soluções dos sistemas acoplado e desacoplados, utilizando três testes, onde fixa-se a advecção e utiliza-se valores diferentes para a difusão. Também, utiliza-se duas discretizações nas malhas, isto é, $\Delta x=0.1 \mathrm{e} \Delta x=1.0 \mathrm{~m}$. Sendo assim, as análises das diferentes formas (AI, DSI e DE) serão observadas considerando o número de Peclet, calculado por $P e=\frac{|u| \Delta x}{2 D}$ [8], como apresentado na Tabela 1 .

Tabela 1: Valores da difusão, da adveção e de Peclet.

\begin{tabular}{|c|c|c|c|}
\hline Parâmetro & Teste 1 & Teste 2 & Teste 3 \\
\hline Difusão (D) & $0.05 \mathrm{~m}^{2} \mathrm{~d}^{-1}$ & $2.5 \mathrm{~m}^{2} \mathrm{~d}^{-1}$ & $25 \mathrm{~m}^{2} \mathrm{~d}^{-1}$ \\
\hline Advecção $(\mathrm{u})$ & $2.5 \mathrm{md}^{-1}$ & $2.5 \mathrm{md}^{-1}$ & $2.5 \mathrm{md}^{-1}$ \\
\hline$P_{e}(\Delta x=0.1)$ & 2.5 & 0.05 & 0.005 \\
\hline$P_{e}(\Delta x=1.0)$ & 25 & 0.5 & 0.05 \\
\hline
\end{tabular}

\subsection{Exemplo envolvendo Reações Irreversível e Reversível}

O exemplo, a ser apresentado, ilustra o transporte de degradação de quatro espécies decomposto em três cadeias de reações, onde duas cadeias procedem em uma única direção, representando as reações irreversível $\left(C_{1} \rightarrow C_{2} \rightarrow\right.$ e $\left.C_{1} \rightarrow C_{3} \rightarrow C_{4} \rightarrow\right)$ e a outra cadeia procede em direções diferentes, representando a reação reversível $\left(C_{2} \leftarrow C_{3} \rightarrow\right)$, como pode ser observado na Figura 1.

O sistema unidimensional de ETAD com reações referente ao esquema apresentadado na Figura 1, pode ser escrito na notação matricial como

$$
\frac{\partial \mathbf{C}}{\partial t}+u \frac{\partial \mathbf{C}}{\partial x}-D \frac{\partial^{2} \mathbf{C}}{\partial x^{2}}=-\mathbf{K C}
$$

sendo

$$
\mathbf{C}=\left[\begin{array}{c}
C_{1} \\
C_{2} \\
C_{3} \\
C_{4}
\end{array}\right], \quad \mathbf{K}=\left[\begin{array}{cccc}
k_{1} & 0 & 0 & 0 \\
-y_{21} k_{1} & k_{2} & -y_{23} k_{3} & 0 \\
-y_{31} k_{1} & 0 & k_{3} & 0 \\
0 & 0 & -y_{43} k_{3} & k_{4}
\end{array}\right]
$$

$C_{1}, C_{2}, C_{3}$ e $C_{4}$ são as concentrações das quatro espécies a serem transportadas, $k_{1}, k_{2}, k_{3}$ e $k_{4}$ são os parâmetros de decaimento de primeira ordem das espécies, $y_{21}, y_{23}, y_{31}$ e $y_{43}$ são os fatores de ganho de uma espécie para a outra.

As concentrações iniciais das espécies são assumidas como sendo nulas e as condições de fronteira como sendo $C_{1}(0, t)=1.0, C_{2}(0, t)=0.0, C_{3}(0, t)=0.0$, 


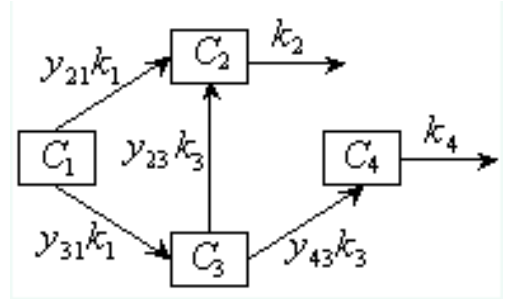

Figura 1: Esquema de reações irreversível e reversível

$C_{4}(0, t)=0.0 \mathrm{mgL}^{-1}$. Os valores dos coeficientes $u$ e $D$ encontram-se descritos na Tabela 1 e quanto aos demais parâmetros, utilizou-se $k_{1}=0.075, k_{2}=0.05$, $k_{3}=0.02, k_{4}=0.045 d^{-1}, y_{21}=0.75, y_{23}=0.2, y_{31}=0.25$ e $y_{43}=0.8$.

Todas as soluções são obtidos após 100 dias de lançamento contínuo em um canal de comprimento de 100 metros, usando dois tipos de malha, uma mais refinada, onde $\Delta x=0.1$ e uma outra com $\Delta x=1.0 \mathrm{~m}$ e a variação no tempo $\Delta t$ igual a 0.5 dias.

Os resultados podem ser observados nas Figuras 2 e 3, verificando que são mostrados apenas os primeiros 30 metros do canal na Figura 2. Estes resultados permitem observar uma boa concordância entre as soluções dos modelos AI, DSI e DE (Testes 1 e 2). Porém, os resultados do modelo DE (Teste 3) apresentaram pequenas diferenças entre as outras soluções dos modelos, observando ainda que tais resultados independem da discretização utilizada.

\section{Conclusão}

Neste trabalho apresentou-se soluções numéricas e analíticas de três formas diferentes de se resolver um sistema de ETAD com decaimento em um meio fluido. Para avaliar a aplicabilidade das formas de soluções acoplada implícita (AI) e desacopladas sequencialmente implícita (DSI) e explícita (DE), realizou-se três testes, onde utilizou-se diferentes valores para a difusão e fixou-se a advecção.

Considerou-se, para os testes apresentados nas Figuras 2 e 3, discretizações diferentes nas malhas, isto é, malhas com $\Delta x=0.1$ e $\Delta x=1.0$, porém pode-se oservar que as discretizações não influenciaram nos resultados.

Se $P e>1$, pode-se observar no Teste 1, apresentado nas Figuras 2 e 3, que os sistemas AI, DSI e DE geram resultados satisfatórios. Também, verificou-se que se $P e<1$, isto é, a adveçcão é menor ou igual a difusão, os resultados do Teste 2 apresentaram pequenas diferenças comparando as soluções das formas AI e DSI com DE, porém, quando o valor da difusão torna-se muito maior que o valor da advecção, Teste 3, estas diferenças aumentam. Assim, pode-se dizer que o aumento da difusão só influência a solução do modelo DE e não os resultados das soluções dos modelos AI e DSI.

As soluções dos modelos AI e DSI, em todos os resultados apresentados nas Figuras 2 e 3 foram obtidas utilizando o método dos elementos finitos (MEF) estabilizados na sua formulação semi-discreta, sujeito a condições iniciais e de fronteira, onde 

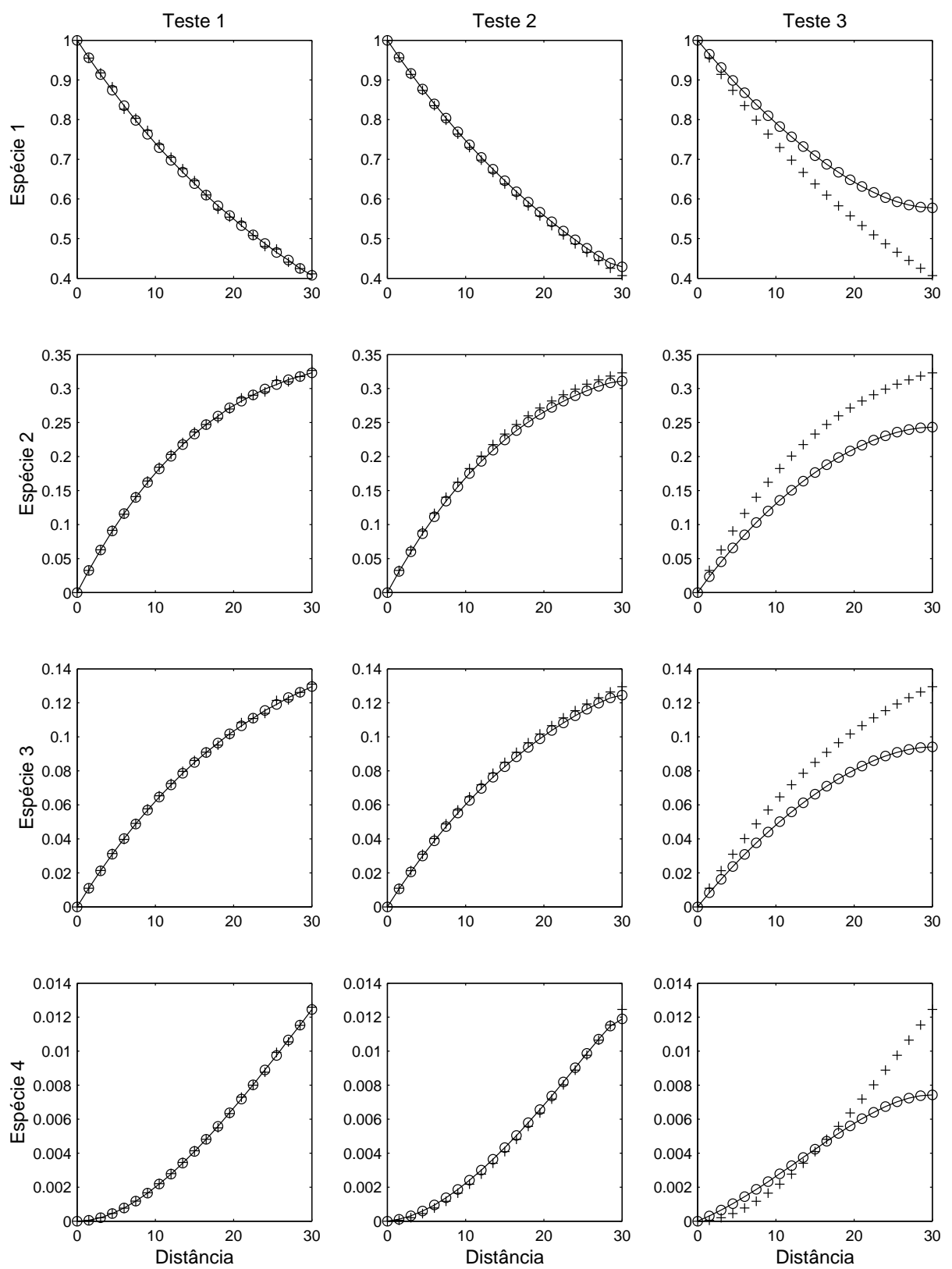

Figura 2: Comparações das formas de soluções: acoplada implícita (oo), desacoplada sequencialmente implícita (linha contínua) e desacoplada explícita $(++)$, usando $\Delta x=0.1 \mathrm{~m}, \Delta t=0.5$ dias e três testes com valores diferentes de difusão. A primeira fila refere-se à espécie $C_{1}$, a segunda à espécie $C_{2}$, a terceira à espécie $C_{3}$ e a quarta à espécie $C_{4}$, respectivamente. 

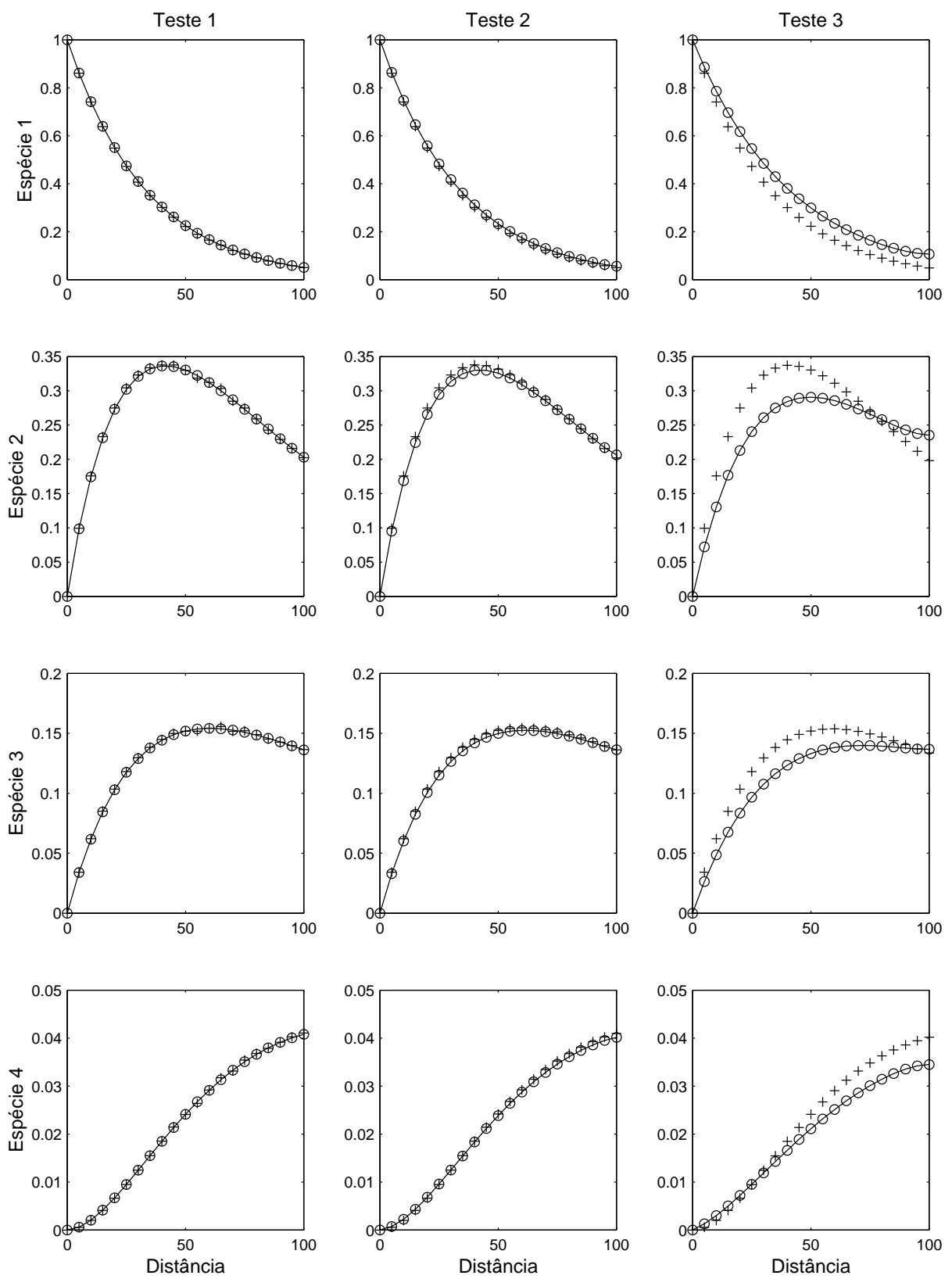

Figura 3: Comparações das formas de soluções: acoplada implícita (oo), desacoplada sequencialmente implícita (linha contínua) e desacoplada explícita $(++)$, usando $\Delta x=1.0 \mathrm{~m}, \Delta t=0.5$ dias e três testes com valores diferentes de difusão. A primeira fila refere-se à espécie $C_{1}$, a segunda à espécie $C_{2}$, a terceira à espécie $C_{3}$ e a quarta à espécie $C_{4}$, respectivamente. 
a variável espacial foi discretizada usando o método dos elementos finitos (SUPG) e a variável temporal, usando o método trapezoidal generalizado Crank-Nicolson. Quanto as soluções do modelo DE, estas foram obtidas resolvendo numericamente a equação diferencial parcial, referente a parte do transporte advectivo-difusivo usando o método dos elementos finitos (SUPG), e resolvendo analiticamente a equação diferencial ordinária, referente a parte das reações.

Conclui-se então, que a forma DE, que é basicamente a solução de um sistema de equações diferenciais ordinárias tendo como condições iniciais a soluções do sistema de equações diferenciais parciais advectivo-difusivo, resulta em algumas restrições quanto aos coeficientes de advecção e difusão.

\begin{abstract}
This work presents an including evaluation of three forms to solve systems of equations of advective-diffusive transport with decay in a media fluid. The forms to be evaluated are differentiated in implicit coupled, implicit sequentially uncoupled and explicit uncoupled. The models can in such a way be described for schematic of reactions irreverível or reversible involving the linear reactions process as the non linear. For the case of the equations that involve non linear processes it can be used the Taylor's expansion in series of first order so as to linearize them, become possible to also analyze this case. One evaluated the gotten results using the Peclet's number calculated. If $P e>1$, where the advection is dominant, the implicit coupled, implicit sequentially uncoupled and explicit uncoupled systems generate satisfactories results. One observes that the results are satisfactory for any Peclet value on the coupled and sequentially uncoupled systems. The same does not occur for the case of the explicit uncoupled, where if $P e<1$, the diffusion is dominant, and the method yield nonsatisfactory results. One evaluated the gotten results considering different values for the diffusion coefficient. It was observed that if the value of the diffusion will be small or equal to the value of the advection the systems implicit coupled, implicit sequentially uncoupled and explicit uncoupled generate satisfactories results. The same it does not occur if the value of the diffusion will be larger that the value of the advection, that in this case the explicit uncoupled generates nonsatisfactory results.
\end{abstract}

\title{
Referências
}

[1] M. Bai, Z. Shu, J. Cao, et al., A semi-analytical solution for a two-dimensional capacitance model in solute transport, Journal of Petroleum Science and Engineering, 22 (1999), 275-295.

[2] A.N. Brooks, T.J.R. Hughes, Streamline-upwind/Petrov-Galerkin formulations for convection dominated flows with particular emphasis on the incompressible Navier-Stokes equations, Comp. Meth. Appl. Mech. Engrg., 32 (1982), 199-259.

[3] M.A. Célia, J.S. Kindred, Contaminant transport and biodegradation 1. A numerical model and test simulations, Water Resources Research, 25, No. 6 (1989a), 1149-1159.

[4] M.A. Célia, J.S. Kindred, I. Herrera, Contaminant transport and biodegradation. 2. Conceptual model for reactive transport in porous media, Water Resources Research, 25, No. 6 (1989b), 1141-1148. 
[5] T.P. Clement, Generalized solution to multispecies transport equations coupled with a first-order reaction network, Water Resources Research, 37, No. 1 (2001), 157-163.

[6] A.E. Croucher, M.J. O'Sullivan, Numerical methods for contaminant transport in river and estuaries, Computers \& Fluids, 27, No. 8 (1998), 861-878.

[7] P.A. Domenico, An analytical model for multidimensional transport of a decaying contaminant species, Journal of Hydrology, 91 (1987), 49-58.

[8] D.A. Farrell, A.D. Woodbury, Numerical modelling of mass transport in hydrogeologic environments: performance comparison of Laplace Transform Galerkin and Arnoldi modal reduction schemes, Adv. Water Resources, 21 (1998), 217-235.

[9] J.J. Kaluarachchi, J. Morshed, Critical assessment of the operator-splitting technique in solving the advection-dispersion-reaction equation: 1. First-order reaction, Advances in Water Resources, 18, No. 2 (1995), 89-100.

[10] K. Park, A.Y. Kuo, Multi-step computation scheme: decoupling kinetic from physical transport in water quality models, Water Research, 30, No. 10 (1996), 2255-2264.

[11] N.M.L. Romeiro, "Simulação Numérica de Modelos de Qualidade de água usando o Método de Elementos Finitos Estabilizados", Tese de Doutorado, COPPE/UFRJ/RJ, 2003.

[12] N.M.L. Romeiro, R.G.S. Castro, L. Landau, "Análise comparativa de modelos de transporte de poluentes com reações cinéticas linear, linearizadas e não linear", em 56º Seminário Brasileiro de Análise, Niterói, RJ, 917-927, 2002.

[13] N.M.L. Romeiro, R.G.S. Castro, L. Landau, "Modelo Acoplado de Transporte de Poluentes de Múltiplas Espécies com Reações Cinéticas Não Lineares", em $58^{\circ}$ Seminário Brasileiro de Análise, UNICAMP, Campinas, SP, 465-472, 2003.

[14] N.M.L. Romeiro, R.G.S. Castro, S.M.C., Malta, L. Landau, "A Linearization Technique for Multi-species Transport Problems", Transport in Porous Media, Publicado online, http://dx.doi.org/10.1007/s11242-006-9081-4, 2007.

[15] Y. Sun, J.N. Petersen, T.P. Clement, et al., Development of analytical solutions for multispecies transport with serial and parallel reactions, Water Resources Research, 35, No. 1 (1999a), 185-190.

[16] Y. Sun, J.N. Petersen, T.P. Clement, Analytical solutions for multiple species reactive transport in multiple dimensions, Journal of Contaminant Hydrology, 35 (1999b), 429- 440.

[17] M.T. Van Genuchten, Analytical solutions for chemical transport with simultaneous adsorption, zero-order production and first-order decay, Journal of Hydrology, 49 (1981), 213-233. 